RIASA TM-88262

NASA Technical Memorandum 88262

NASA-TM-88262 19860018616

\title{
Test and Evaluation of the HIDEC Engine Uptrim Algorithm
}

\section{Ronald J. Ray and Lawrence P. Myers}

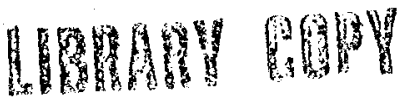

$\because \quad 8,8 j$

GANGLEY FESTARCH CENTER

IIGRARY, NASA

MAnOPTON, VIRGINIA 


\section{Test and Evaluation of the HIDEC Engine Uptrim Algorithm}

Ronald J. Ray and Lawrence P. Myers

Ames Research Center, Dryden Flight Research Facility, Edwards, California

\section{N/SA}

National Aeronautics and

Space Administration

Ames Research Center

Dryden Flight Research Facility

Edwards, California 93523-5000 
TEST AND EVALUATION OF THE HIDEC ENGINE UPTRIM ALGORITHM

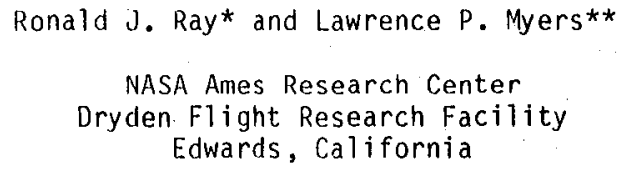

\section{Abstract}

The highly integrated digital electronic control (HIDEC) program will demonstrate and evalwate the improvements in performance and mission effectiveness that result from integrated engineairframe control systems. Performance improvements will result from an adaptive engine stall margin mode, a highly integrated mode that uses the airplane flight conditions and the resulting inlet distortion to continuously compute engine stall margin. When there is excessive stall margin, the engine is uptrimmed for more thrust by increasing engine pressure ratio (EPR).

The EPR uptrim logic has been evaluated and implemented into computer simulations. Thrust improvements over 10 percent are predicted for subsonic flight conditions. The EPR uptrim was successfully demonstrated during engine ground tests. Test results verify model predictions at the conditions tested.

\section{Nomenclature}

ABSEUM augmenter sequencing uptrim margin

ADECS adaptive engine control system

ALPHA angle of attack, deg

BETA sideslip angle, deg

CAS control augmentation system

DEEC digital electronic engine control

DEFCS digital electronic flight control system

DEPRLIM DEEC maximum EPR limit

DFCC digital flight control computer

DFPR change in fan pressure ratio

EMD engine model derivative

EPR engine pressure ratio, PT6/PT2

EPRN engine pressure ratio nominal schedule

EPRP engine pressure ratio for optimum performance

EPRS engine pressure ratio for maximum stability (minimum inlet distortion)

EPRUS engine pressure ratio for maximum stability (adjusted to actual inlet distortion)

*Aerospace Engineer.

**Aerospace Engineer. Member AIAA.

EPRUT EPR uptrim command implemented by DEEC

FN

FPR

FPRREF

FPRS

FPRUS

FTIT

HIDEC

KA2

KA2MIN

KA2BASE

KEPR

N1C2 corrected fan speed, rpm

PCEPRP

PCEPRU

PCEPRUS

PLA

PS2

PT2

PT2.5

PT6

SML

UARIT

WACC obtained by integrating flight and propulsion

fan pressure ratio for maximum stability (adjusted to actual inlet distortion)

fan turbine inlet temperature, ${ }^{\circ} \mathrm{F}$

highly integrated digital electronic control

fan distortion factor

minimum fan distortion produced by inlet

fan distortion corrected for angle of attack only

multiplier on EPR command sent to engine DEEC

precentage EPR uptrim based on optimum performanee

percentage EPR command to the DEEC

percentage EPR uptrim based on stability

power lever angle, deg

static pressure at fan inlet, $1 \mathrm{~b} / \mathrm{in}^{2}$

total pressure at fan inlet, lb/in?

total pressure at fan exit, $1 \mathrm{~b} / \mathrm{in}^{2}$

total pressure at turbine, $1 \mathrm{~b} / \mathrm{in}^{2}$

fan stall margin, percent

universal asynchronous receivertransmitter

corrected fan airflow, 1b/sec

\section{Introduction}

Substantial performance benefits can be

obtained by integrating flight and propulsion 
controls. Independent optimization of each control system is usually compromised by worst-case assumptions regarding other systems. Integration al lows the control systems to work together.

In current-technology fighter engines, the fan operating line is scheduled to avoid stall, based on a statistical worst-case assessment of each destabilizing effect. These effects include altitude or Reynolds number variation, engine-toengine and control tolerance variations, augmenter sequencing, hack-pressure spikes, and inlet distortion.l An additional safety margin (remaining stall margin) is generally built in.

In the adaptive engine control system (ADECS) mode, the stali margin required for distortion and for augmenter sequencing and the remaining stall margin will be available for additional engine pressure ratio (EPR) uptrim. During flight conditions in which distortion is low, the stall margin required for distortion effects will be reduced to a minimum.

An evaluation of the highly integrated digital electronic control (HIDEC) system is cur rently being completed at the Dryden Flight Research Facility of NASA's Ames Research Center (Ames-Dryden).2 A digital electronic engine control (DEEC) is installed on an F100 engine model derivative (EMD) engine in the Ames-Dryden F-15 research airplane. The DEEC is a full-authority, single-channel digital control with an integral hydro-mechanical backup control. A digital electronic flight control system (DEFCS), which will accommodate the HIDEC computations, is also installed in the airplane. The various diggital systems on the airplane can communicate with each other through a digital interface and bus controller.

HIDEC methodology has been established for scheduling the optimum fan match in response to inlet distortion. This has been defined in detail for the F100 EMD engine by extensive steady-state model testing. A stability limit based on the minimum stall margin has been developed using a stability audit. The optimum-performance uptrim has been established based on thrust gains using steady-state simulation predictions. The ADECS procedure allows an onboard aircraft computer to estimate the level of inlet distortion and select the proper uptrim request to send to the engine control. The DEEC responds by closing the nozzle until EPR increases to the requested value. The net result is increased thrust.

This paper will describe the HIDEC system, EPR uptrin methodology and logic, computer simulations, and ground test results.

\section{Airplane}

The NASA F-15 research airplane at Ames-Dryden is being used for the HIDEC program. The F-15 is a high-performance air superiority fighter with excellent transonic maneuverability and a maximum Mach capability of 2.5. It is powered by two afterburning turbofan engines.
Engine

The F100 EMD engine (Pratt and Whitney PW1128) (Fig. 1) is an upgraded version of the F100-PW-100 engine that currently powers the production $F-15$ airplanes. The engine incorporates a redesigned fan, revised compressor and combustor, singlecrystal turbine blades and vanes, a 16-segment augmenter with light-off detector, and a DEEC.

The DEEC, a key part of the HIDEC system, 3,4 is a full-authority digital control with an integral hydromechanical backup control. It controls the gas generator and augmenter fuel flows, the compressor bleeds, the variable inlet guide vanes, the variable stators, and the variable exhaust nozzle. The DEEC incorporates logic that provides closed-loop control of engine airflow (WACC) and EPR. It also limits fan turbine inlet temperature (FTIT). It has the capability of accepting inputs, from the airplane as well as from the many engine sensors.

\section{HIDEC System}

The equipment that is installed on the F-15 airplane for the HIDEC program (shown in Fig. 2) includes the F100 EMD engines, currently involved in testing at Ames-Dryden, along with the DEEC. engine controllers; a digital electronic flight control system, which will accommodate the HIDEC computations; a digital interface and bus control unit; and a cockpit control and display. A telemetry uplink from ground-based computers is also available. The F-15 airplane is fully instrumented and equipped for propulsion and flight control integration research.

A block diagram of the F-15 HIDEC system is shown in Fig. 3. The various digital systems on the airplane will communicate with each other through a digital interface and bus controller. This unit will permit the HIDEC system to communicate with the equipment on the F-15 H009 data bus, the universal asychronous receivertransmitter (UART) data bus from the DEEC, and the 1553 flight control data bus.

The DEEC controllers on each engine will communicate with the HIDEC system through the UART bus. The normal throttle inputs to the DEEC controllers and the backup engine controls from the cockpit will be maintained. Additional information on the HIDEC system can be found in Ref. 5 .

\section{Engine Pressure Ratio Uptrim Logic}

The EPR uptrim logic resides in the digital flight control computer. (DFCC) and determines the proper EPR command to send to the DEEC to obtain higher thrust. To accomplish this, two EPRs are calculated: the optimum-performance E.PR and the limiting stability EPR. The lower value is selected as the HIDEC uptrim EPR. This command, expressed as a percentage, is passed to the DEEC where it is checked for validity against the DEEC EPR limit. Figure 4 illustrates the HIDEC EPR uptrim schedules on a typical fan map. The stall line is shown as the upper limit. The distance 
between the stall line and the normal operating line represents the stall margin available. The DEEC EPR limit represents the maximum EPR allowed by the DEEC during uptrim. The stability EPR takes into consideration al1 destabilizing factors that reduce the fan stall margin from the basic steadystate level. As inlet distortion increases, the stability EPR adjusts downward to maintain an adequate stall margin. The optimum-performance EPR does not take stall margin into account; it represents the optimum EPR based on predicted thrust gains.

Figure 4 shows one hypothetical uptrim case. Point $N$ represents the normal operating point. An uptrim request first increases EPR at a constant corrected fan speed until the FTIT limit is reached (point $T$ ). The EPR then continues to increase along the FIIT limit until the stability EPR is reached (point $S$ ). This is the limiting. factor for this case and is based on predicted inlet distortion. To maintain an adequate stall margin, no additional EPR increases are allowed. Point $D$ represents the EPR limit scheduled in the DEEC; this limit protects against unreasonably large uptrim requests. The DEEC EPR limit is slightly higher then the stability EPR during conditions of minimum distortion. The optimumperformance EPR (point $P$ ) is not attainable in. this case, because it is too close to stall (point ST) and would not allow an adequate remaining stall margin. As shown in the figure, the optimum-performance schedule is only attainable at the lower fan speeds for the case presented.

The DEEC responds to the uptrim request by closing the nozzle until the desired EPR is reached. The increase in EPR results in an increase in engine thrust. The uptrim logic is limited to power settings at power lever angles (PLA) greater than $70^{\circ}$. When uptrim is requested, the DEEC slews in the uptrim value over a period of $1 \mathrm{sec}$. This allows for a smooth transition to. uptrim operation.

Figure 5 is a flow diagram of the EPR uptrim logic located in the digital flight control computer. The blocks illustrate schedules and calculations; the arrows denote data flow.

Reference 6 describes the methodology and results of a study to determine the maximum allowable EPR uptrim for the PW1128 engine at minimum levels of inlet distortion.

\section{Optimum-Performance Engine Pressure Ratio}

The optimum-performance EPR (EPRP) represents that EPR at which optimum engine thrust will be obtained. It is scheduled as a function of corrected fan speed (N1C2) and inlet total pressure (PT2), as shown in box 1 of Fig. 5. The schedule was determined from extensive simulation data obtained by using the steady-state engine simulation at a variety of flight conditions; the EPR was increased incrementally at each condition until the peak thrust was reached. This peak thrust corresponds to the optimum-performance EPR.
Although the optimum-performance EPR is the most desirable in terms of engine performance, it is oftern unattainable because of stability considerations and protective limits in the DEEC.

\section{Stability Engine Pressure Ratio}

An EPR based on engine stability is calculated to limit the amount of uptrim during conditions of reduced fan stall margin resulting from inlet distortion. Inlet distortion increases at low Mach numbers, reduced inlet airflows, and elevated angles of attack or sideslip. Because of this complex relationship, the uptrim stability loyic requires the input of both aircraft and engine parameters to calculate the stability EPR limit.

The stability logic begins by calculating the inlet distortion factor (KA2) based on aircraft and engine conditions. A base inlet distortion factor (KA2BASE) is calculated for the current Mach number and predicted angle of attack. This base value is then corrected for the effects of sideslip and engine airflow. These schedules are shown in blocks 2 and 3 of Fig. 5.

The fan pressure ratio (FPR) change (DFPR), based on the stall margin sensitivity to the calculated distortion factor, is calculated next. First, the difference between the calculated inlet distortion factor and the scheduled minimum inlet distortion factor (KA2MIN) is determined; the minimum value is scheduled from the current flight Mach number, as shown in block 4. This differential value of inlet distortion is then multiplied by the stall margin sensitivity factor (block 5 ) to determine the current sensitivity of stall margin to distortion. This result is then multiplied by the scheduled reference value of FPR (FPRREF) in block 6 .

The stability logic uses the change in FPR to adjust the maximum-stability FPR (FPRS), which is computed from the maximum-stability EPR (EPRS), as shown in blocks 7 and 8 . The resulting difference between the maximum-stability FPR and the change in FPR yields the adjusted maximum-stability FPR (FPRUS). This value is then converted back to an EPR. The result is the maximum stability EPR (EPRUS), which is based on actual inlet distortion. The uptrim EPR cannot exceed this value.

\section{Engine Pressure Ratio Limit Logic}

Protective logic resides in the DEEC to protect against erroneous or out-of-range uptrim requests. Figure 6 illustrates the DEEC uptrin limit logic. A maximum EPR limit is compared with the uptrim EPR command (PCEPRU). The DEEC calcillates this maximum EPR limit (DEPRLIM) from inlet total pressure and corrected fan speed. The limiting EPR is based on minimum distortion and was developed from stability audits at numerous flight conditions. The logic incorporates a downtrim to provide for additional stall margin during auymenter sequencing (augmenter sequencing uptriin margin, ABSEUM) and is a function of inlet total pressure only. In most cases this downtrim is 6 percent, except at very low inlet total pressure 
where it can be as much as 10 percent. The logic decides if the augmenter is sequencing; if the augmenter is sequencing, a minimum is selected between the maximum EPR limit and the uptrim EPR cominand, and then the 6 percent downtrim is subtracted. At steady-state maximum power, the downtrin is washed out, and the full uptrim is used. At intermediate (military) and maximum power settings, the logic selects the minimum between the maximum EPR limit and the uptrim EPR command and uses this minimum command (EPRUT) to operate the engine.

\section{Simulation Programs}

Two computer simulations of the Flo0 EMD engine are being used in the HIDEC program: a full aerothermal steady-state engine performance program and a linear state-variable dynamic engine model.

The steady-state model 7 was modified to include the HIDEC EPR uptrim logic. This simulation provides accurate values for many engine parameters including engine thrust, fuel flow, fan and core stall margins, and the DEEC parameters. Its inputs are altitude, Mach number, power lever angle, and uptrim request. An EPR uptrim can be evaluated using the uptrim logic (Fig. 5) or input manually as desired. Options are also available to adjust FTIT, airflow, fan and core efficiencies, and installation effects. The model was derived from actual test data, primarily along the normal operating line.

The dynamic engine mode ${ }^{8}$ was also modified to include the EPR uptrim logic. This simulation. provides realistic dynamic response characteristics for engine transients. It is a piecewiselinear state-variable model that simulates both the engine and the DEEC. Control actuator and sensor dynamics are simulated, and low and high compressor stall margins are calculated. Input requiremients for the dynamic model are similar to those of the steady-state model but are time dependent. The model was derived from a nonlinear aerothermodynamic model and is not as accurate as the steady-state model for stabilized conditions.

\section{Predicted Simulation Results}

Steady-state simulation predictions were made using the F100 EMD engine status model with the HIOEC uptrim logic implemented. ${ }^{9}$ Figure 7 illustrates the effect that angle of attack (ALPHA) (and thus increasing distortion) has on the uptrim logic. Shown are the performance EPR, the stability. EPR, and the maximum EPR limit in the DEEC for the given flight condition. The uptrim logic will select the minimum optimum-performance EPR between $0^{\circ}$ and $.4 .5^{\circ}$ angle of attack and will select the stability EPR for all other angles of attack. The stability EPR drops as the angle of attack moves away from the nominal value because of the increase in inlet distortion. This insures that adequate stall margin is maintained during angleof -attack variations while in the uptrim mode.

The steady-state simulation was used to generate the uptrim envelope shown in Fig. 8 , which illustrates the predicted regions where optimum performance, stability limitations, and no uptrim will occur. The stability-limited region occurs in the upper left-hand corner of the flight envelope, as one would expect, because of higher inlet distortion. At higher Mach numbers, thrust is less sensitive to EPR uptrim as mass flow becomes the dominant factor in the thrust equation. Therefore, minimal performance gains were predicted at higher Mach numbers.

The predicted installed thrust gains for intermediate power are shown in Fig. 9, which illustrates contours of increased net propulsive thrust due to uptrimming at steady-state flight conditions. The greatest performance gains occur at subsonic speeds at altitudes between 10,000 and $40,000 \mathrm{ft}$. Thrust increases of 8 to 10 percent are predicted for this region. Most of the region of maximum thrust gain takes place where the uptrim logic is stability limited, as shown in Fig. 8.

\section{Engine Test}

A sea level engine test of the HIDEC system was conducted to evaluate the ADECS control logic and to determine the performance improvements. 10 The engine was mounted on an outside test stand with a bellmouth inlet. Thrust was measured with a load cell. In flight, the aircraft digital flight control computer calculates EPR uptrim for stability and performance, based on the distortion. This uptrim command is sent to the DEEC through the UART data bus. For the ground test, a digital flight control computer emulator was used to supply the DEEC with uptrim requests.

\section{Steady-State Test Results}

Steady-state uptrim data were obtained at a variety of throttle settings and percentage uptrim requests. Tests without uptrim were also conducted so comparisons could be made between baseline and uptrim cases.

Steady-state data were evaluated by incrementally increasing uptrim EPR at intermediate and maximum power. Figure 10 illustrates the maximum power results along with simulation predictions. The figure shows fan stall margin, FTIT, and thrust increase for uptrim requests up to 12 percent. For this case, EPR increased at a constant fan speed until the FTIT limit was reached. Uptrim then continued along the FTII limit while fan speed decreased, similar to the case illustrated in Fig. 4. Thrust increased up to the point where the FTIT limit was reached. Beyond that, fan speed and airflow decreased, causing thrust to decrease. A maximum 4-percent thrust gain was obtained at 6-percent EPR uptrim. Fan stall margin decreased with uptrim and was 24 percent at the point of maxinum thrust gain, adequate for normal operation. The ADECS mode demonstrates the tradeoff between increased performance and reduced stall margin.

Figure 10 also shows the optimum-performance EPR implemented by the HIDEC control laws. This was calculated with the simulation model using the EPR uptrim logic presented in Fig. 5. As shown, 
the optimum-performance EPR adequately represents the proper EPR for optimum thrust for this case.

The intermediate power incremental uptrim test results are shown in Fig. 11. The baseline FTIT was lower for this case than at maximum power because at maximum power the engine must make up for the turbopump bleed extraction required to drive the augmenter fuel pump, resulting in a high FTIT. Because the baseline FTIT is lower at intermediate power, more EPR uptrim is required to reach the FTIT limit. As in the maximum power case, thrust increased until the FTIT limit was reached, then decreased because of reduced airflow. At 10-percent EPR uptrin, a 7-percent thrust increase was demonstriated. The stall margin decreased with uptrim, as expected, and was 22 percent at the maximum thrust gain demonstrated. This is adequate for normal engine operation.

The HIDEC uptrim EPR implemented by the control laws is also shown for this case. Because uptrim EPR is scheduled as a function of inlet total pressure and corrected fan speed, it is the same value as in the maximum power case. For engine safety reasons, uptrim EPR was derived from a conservative standpoint. It does not exceed the peak performance gain for either intermediate or maximum power cases, beyond which performance decreases and stall margin diminishes. For the two power settings demonstrated, maximum power was the limiting case. The intermediate power case is therefore compromised as shown. In later testing, separate maximum and intermediate power uptrims may be needed.

Simulation predictions are in excellent agreement with the results presented in Figs. 10 and 11 until the maximum performance gain is reached. Beyond this point, the simulation begins to deviate from the test results because of the simulation's limited capability to model the engine at extreme off-nominal conditions, such as large uptrim request.

A comparison of measured and predicted EPR and thrust for 7-precent uptrim at varlous power settings is shown in Fig. 12. Test results confirm that the uptrim logic prevents upmatch below $70^{\circ}$ power lever angle. Uptrim was smoothly implemented at power settings above $70^{\circ}$ and was successfully downtrimmed 6 percent during augmenter sequencing. The figure shows substantial thrust gains due to uptrim at intermediate and maximum power. Simulation results for the uptrim case predict a slight bias (2 percent low) for most power settings. At maximum power, the EPR prediction is slightly higher. Thrust predictions agree with test results, except in the maximum power case where the simulation predicts higher thrust, which corresponds to the higher EPR prediction.

Dynamic. Performance Results

During dynamic performance tests, augmenter transients were demonstrated with EPR uptrim. The 6 -percent downmatch (during augmenter sequencing) was successfully demonstrated (Fig. 13). The sequencing downmatch provides additional stall margin for augmenter pressure spikes caused by augmenter ignition and segment sequencing. For comparison, both baseline and uptrin cases are shown along with the predicted results from the engine dynamic model. The downmatch occurred after snapping the throttle from intermediate to maximum power. After augmenter sequencing was completed and the engine reached maximum power, the sequencing downmatch was washed out. The simulation predicts an unreasonable pressure spike for both the baseline and the uptrimmed case shortly after advancing the throttle, perhaps because of nozzle area modeling errors. For the uptrim case, the simulation differs from the actual EPR, but it does follow the same trend. The dynamic model is not as accurate as the steadystate program and also suffers the same limitations while trying to model the engine during off-scheduled operations, such as uptrimming.

\section{Concluding Remarks}

The HIDEC EPR uptrim logic was successfully demonstrated and evaluated during engine ground testing. Thrust increases of 4 and 7 percent were demonstrated at maximum and intermediate power settings, respectively. The 6-percent downmatch during augmenter sequencing was also demonstrated. Engine. simulation results compare favorably with test results, lending confidence to predictions.

\section{References}

lYonke, W.A., Terrel1, L.A., and Meyers, L.P., "Integrated Flight/Propulsion Control: Adaptive Engine Control System Mode," AIAA$85-1425$, July 1985.

2Myers, Lawrence P., and Burcham, Frank W., Jr., "Propulsion Control Experience Used in the Highly Integrated Digital Electronic Control (HIDEC) Program," NASA TM-85914, 1984.

3Burcham, Frank W., Jr., Myers, Lawrence P., and Walsh, Kevin R., "Flight Evaluation Results for a Digital Control System in an F-15 Airplane," NASA TM-84918, 1983.

4Barrett, W.J., Rembold, J.P., Burcham, F.W., and Myers, L., "Flight Test of a Full Authority Digital Electronic Control System in an F-15 Airplane," AIAA-81-1501, July 1981.

5Burcham, Frank W., Jr., and Haering, Edward A., Jr., "Highly Integrated Digital Engine Control System on an F-15 Airolane," NASA TM-86040, 1984.

6Kelly, J.B., and Yuhas, A.J., "PWI128 HIDEC EPR Uptrim Limits Final Report," Pratt and Whitney Report FR-18852, Jan. 1985.

7"F100 EMDP Prototype Engine Flight Demonstration Performance Model, User's Manual for Deck CCD 1194-4.0," Pratt and Whitney Report FR-16212, Mar. 1983.

80gburn, Stephen, "Computer Simulation User's Manual for CCD 1246-01.0, State Variable Dynamic Model of PW 1128 With a DEEC Control," Pratt and Whitney Report FR-18761A, May 1985. 


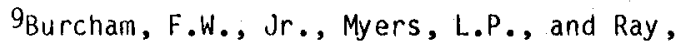
R.J., "Predicted Performance Benefits of an Adaptive Digital Engine Control System on an F-15 Airplane," AIAA-85-0255, Jan. 1985.
10Andries, M.G., and Terrel1, L.A., "Highly Integrated Digital Electronic Control HIDEC Sea Level Test Report for Engine P085-11," Pratt and Whitney Report FR-19248, Jan. 1986.

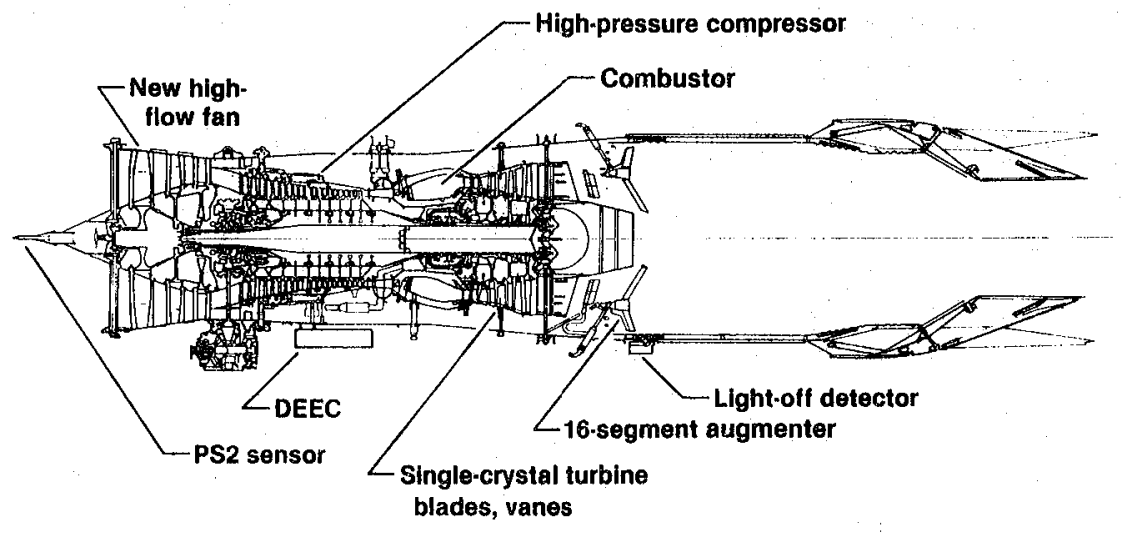

Fig. 1 Features of the F100 EMD engine for the HIDEC program.

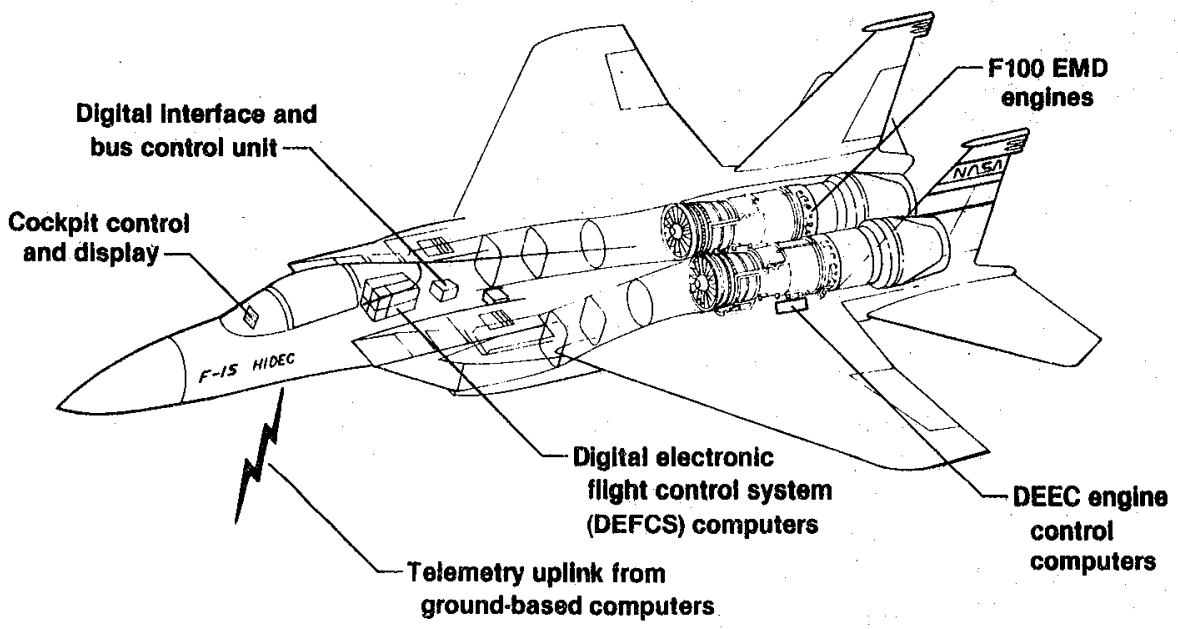

Fig. 2 Features of the F-15 HIDEC research airplane. 


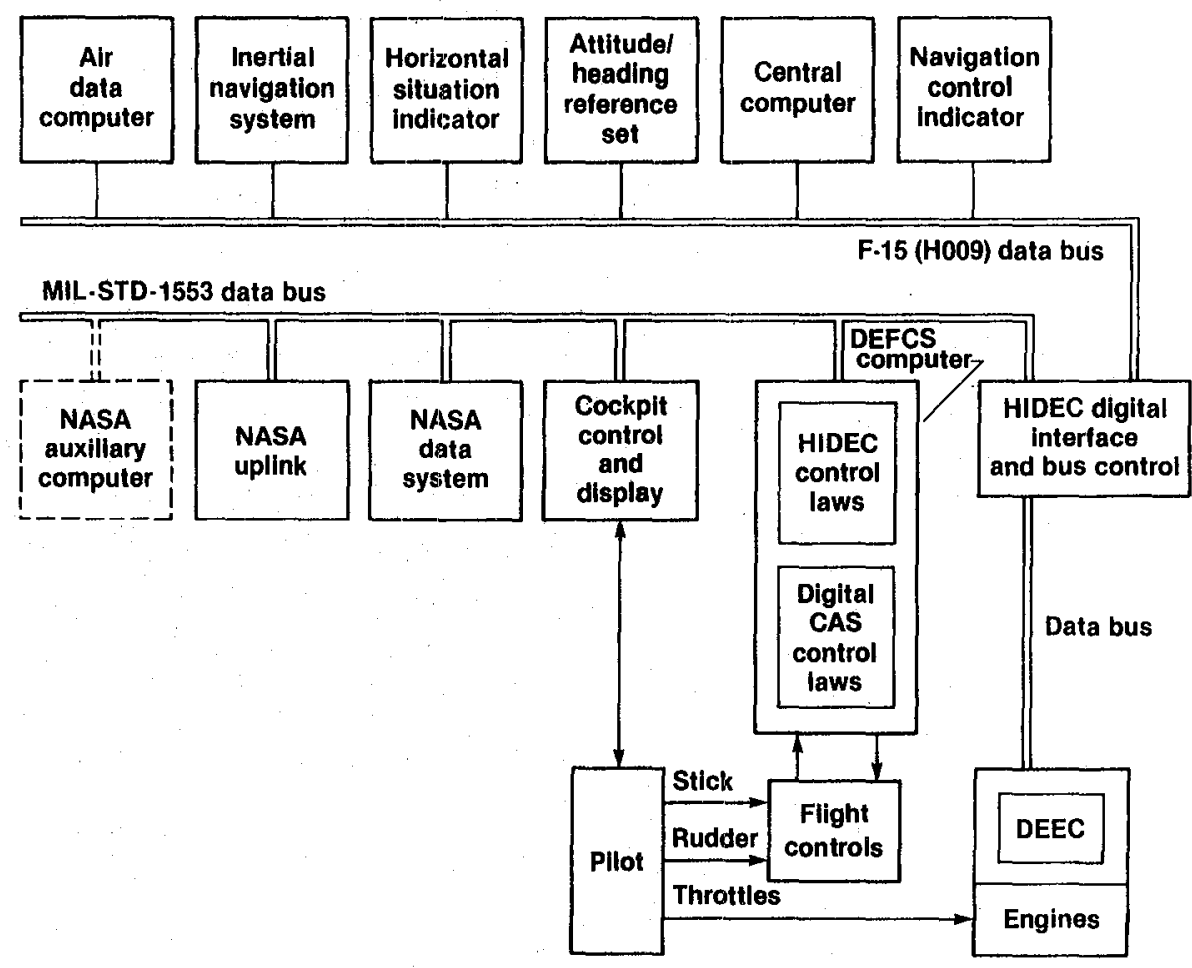

Fig. 3 Block diagram of the HIDEC system.

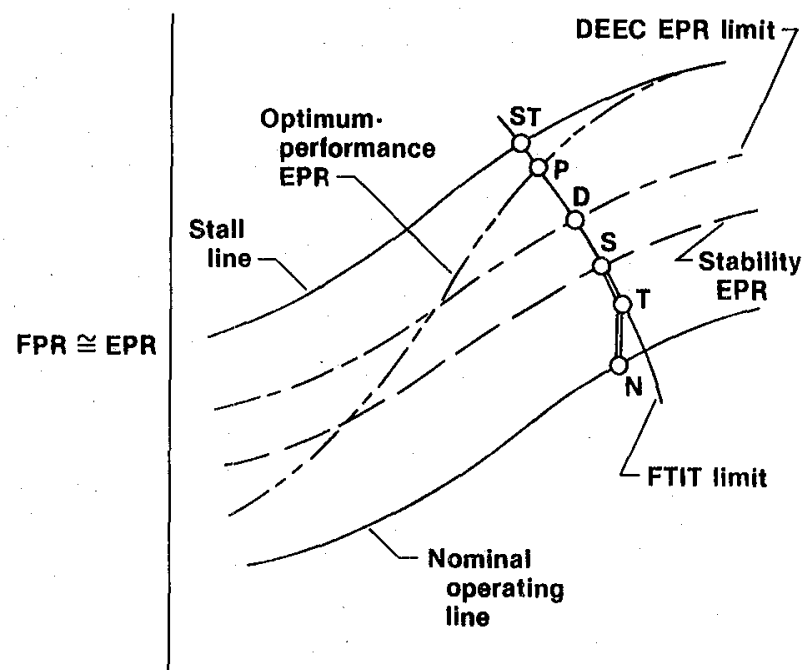

Corrected fan speed, N1C2

Fig. 4 HIDEC EPR uptrim schedules. 

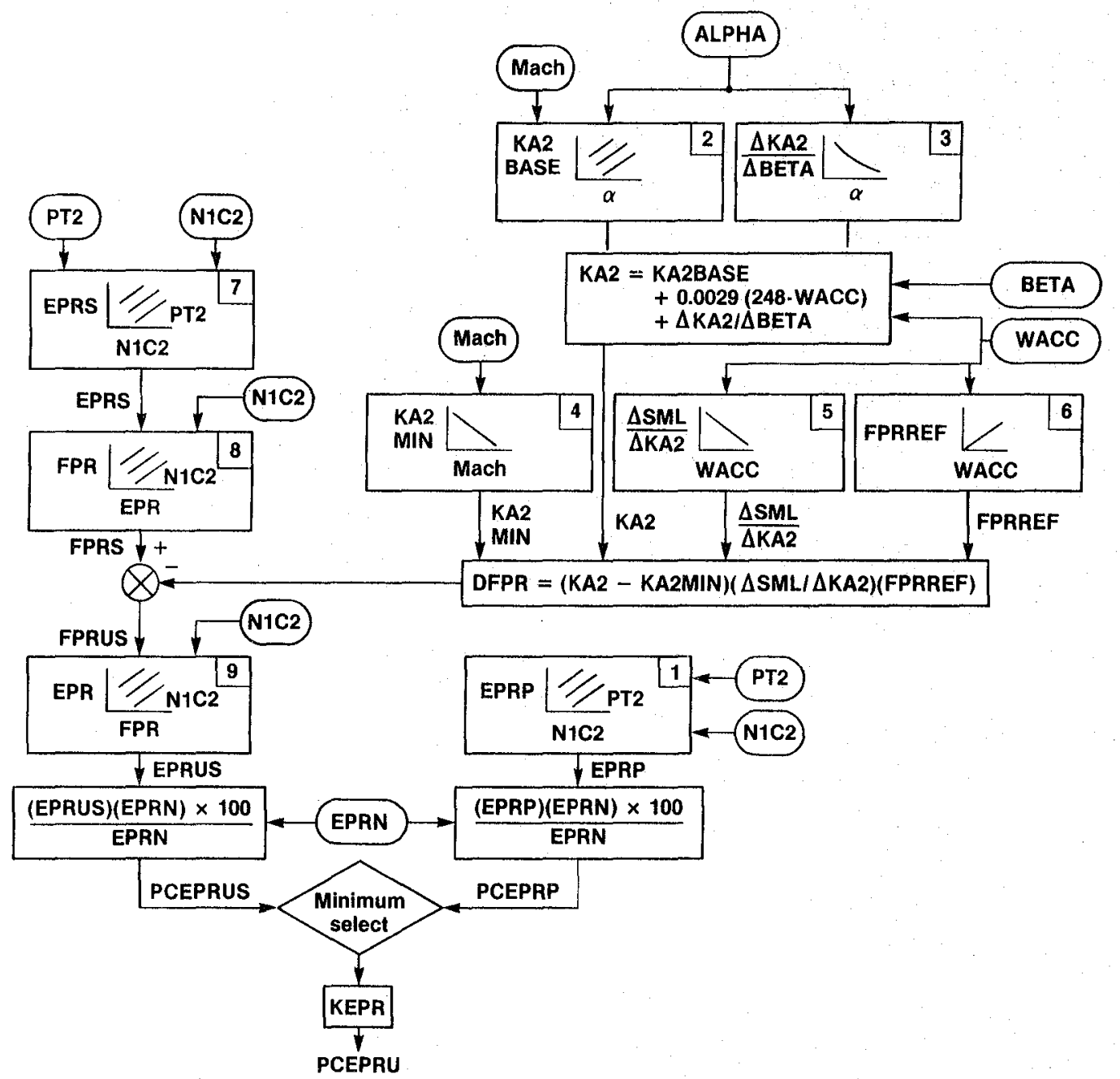

Fig. 5 Flow diagram of EPR uptrim logic.

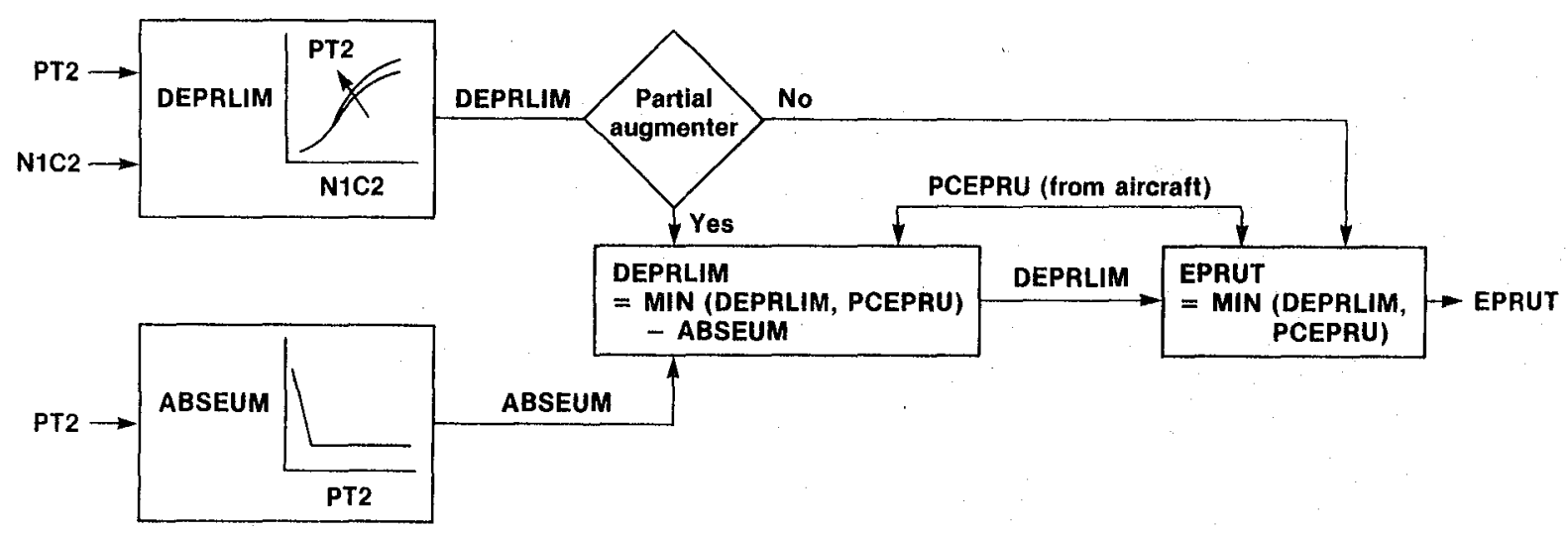

Fig. 6 DEEC uptrim limit logic. 


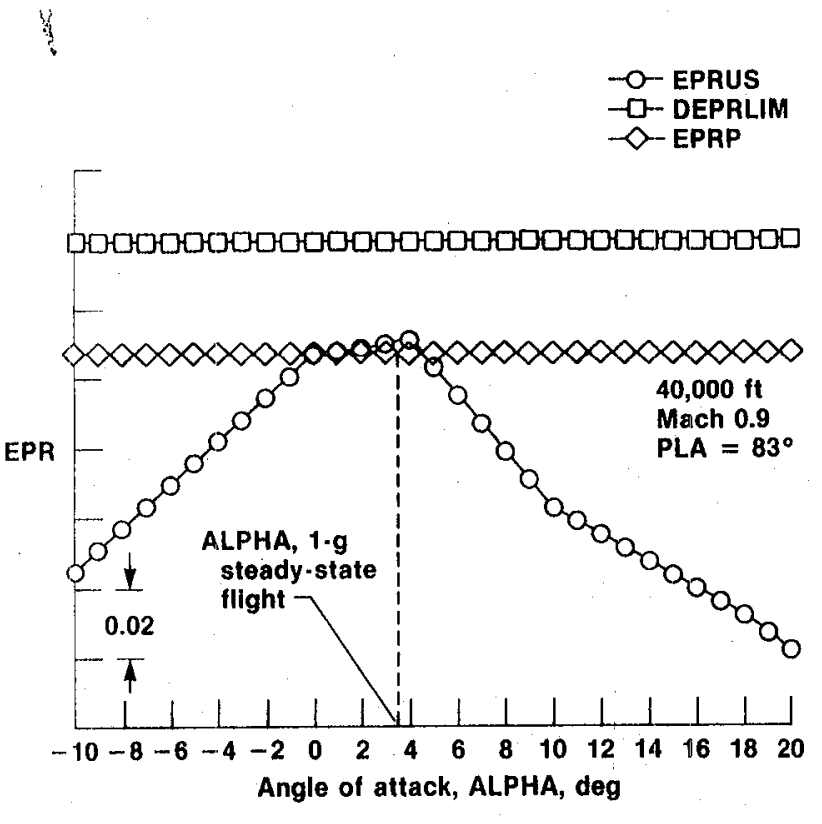

Fig. 7 Effeot of angle of attack on uptrim logic.

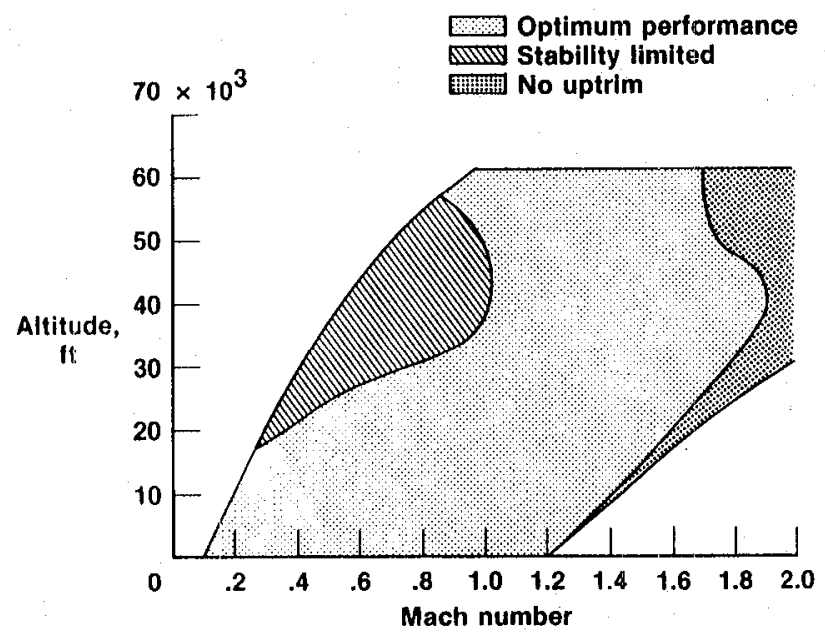

Fig. 8 HIDEC uptrim envelope, intermediate power settings.

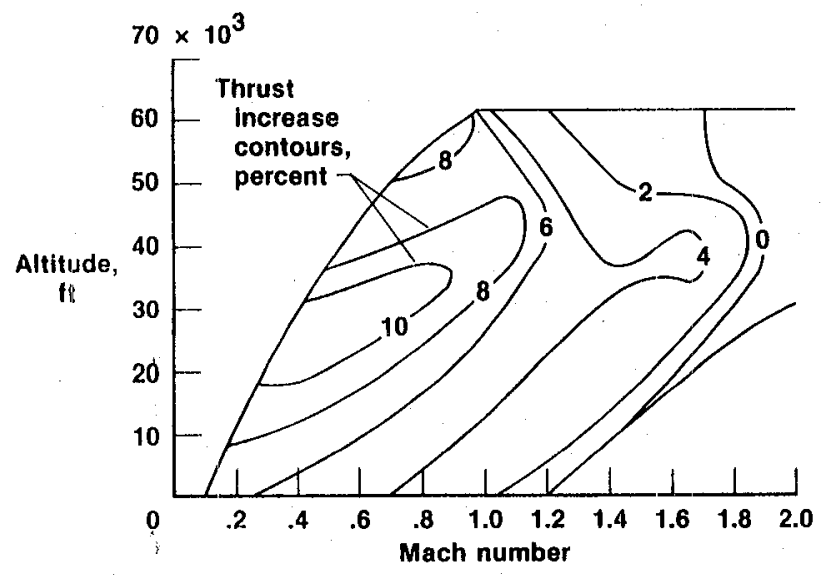

Fig. 9 Fredicted intermediate power thmust gains. 

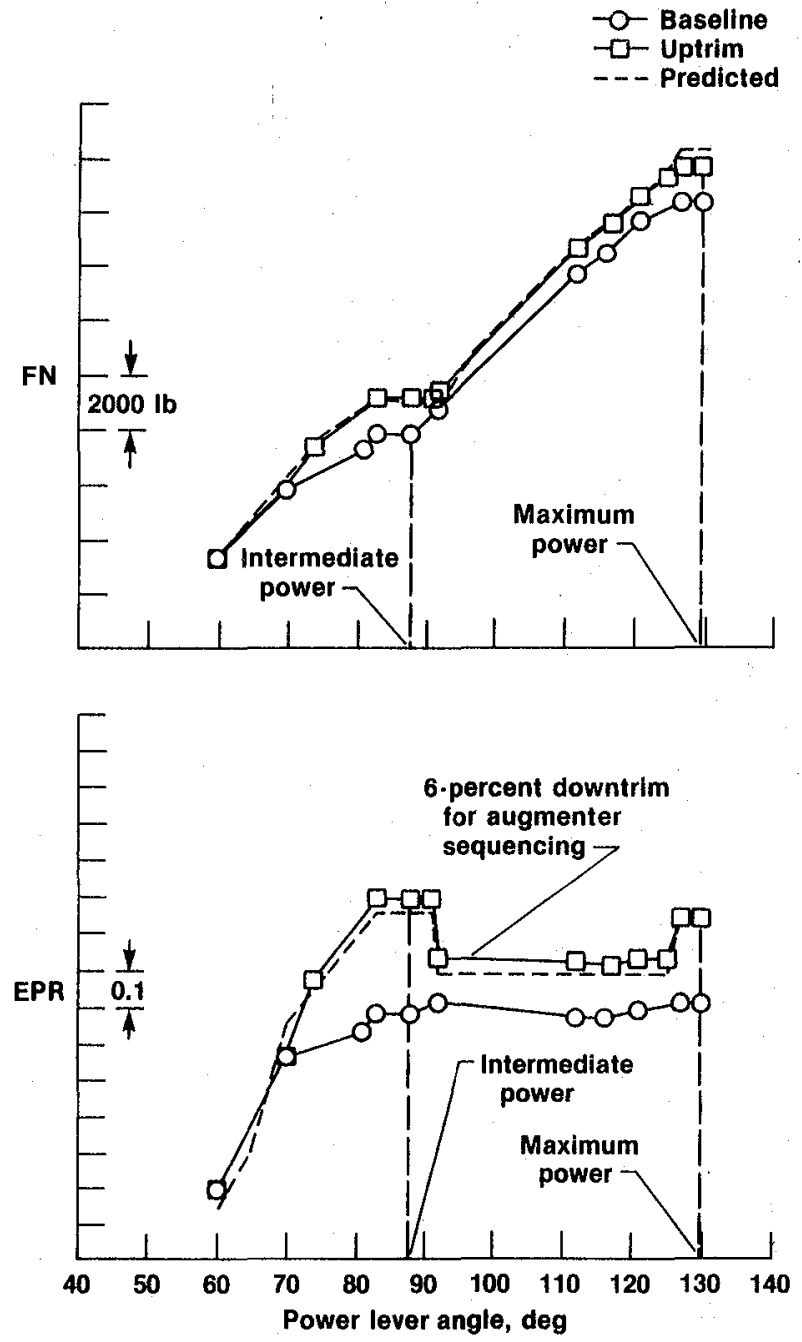

Fig. 12 Comparison of measured and predicted EPR and thrust, sea level static for a 7-percent uptrim.

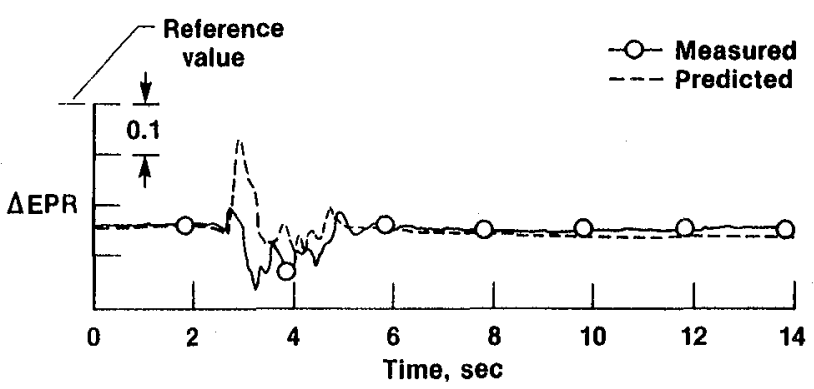

(a) Intermediate-maximum throttle transient without uptrim.

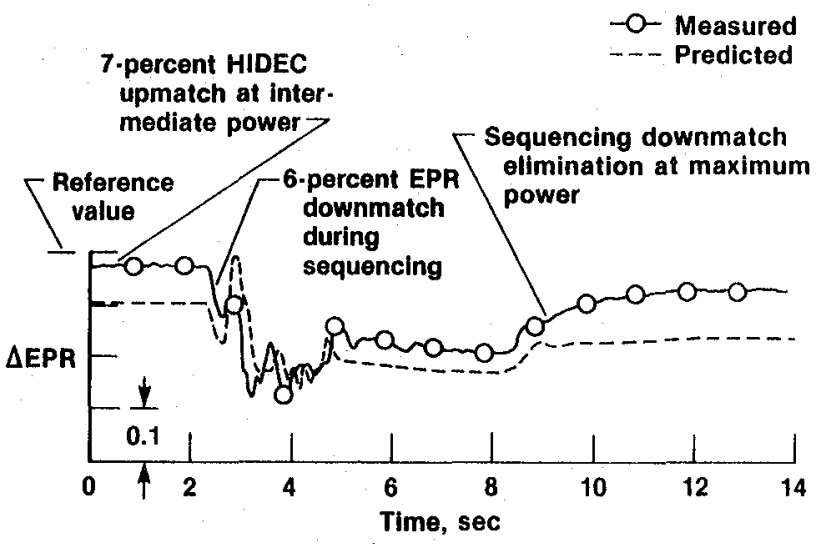

(b) Intermediate-maximum throttle transient with uptrim.

Fig. 13 Evaluation of the uptrim logic during augmenter operation. 




*For sale by the National Technical Information Service, springfield, Virginia 22161. 
End of Document 